\title{
Pseudo-stokes vector from complex signal representation of a speckle pattern and its
} applications to micro-displacement measurement

Wang, W.; Ishijima, R.; Matsuda, A.; Hanson, Steen Grüner; Takeda, M.

Published in:

Strain

Link to article, DOI:

10.1111/j.1475-1305.2009.00672.x

Publication date:

2010

Document Version

Publisher's PDF, also known as Version of record

Link back to DTU Orbit

Citation (APA):

Wang, W., Ishijima, R., Matsuda, A., Hanson, S. G., \& Takeda, M. (2010). Pseudo-stokes vector from complex signal representation of a speckle pattern and its applications to micro-displacement measurement. Strain, 46(1), 12-18. https://doi.org/10.1111/j.1475-1305.2009.00672.x

\section{General rights}

Copyright and moral rights for the publications made accessible in the public portal are retained by the authors and/or other copyright owners and it is a condition of accessing publications that users recognise and abide by the legal requirements associated with these rights.

- Users may download and print one copy of any publication from the public portal for the purpose of private study or research.

- You may not further distribute the material or use it for any profit-making activity or commercial gain

- You may freely distribute the URL identifying the publication in the public portal 


\title{
Pseudo-Stokes Vector Correlation From Complex Signal Representation of a Speckle Pattern and Its Applications to Micro-Displacement Measurement
}

\author{
W. Wang*, R. Ishijima ${ }^{\dagger}$, A. Matsuda ${ }^{\dagger}$, S. G. Hanson ${ }^{\ddagger}$ and M. Takeda ${ }^{\dagger}$ \\ *Department of Mechanical Engineering, School of Engineering and Physical Sciences, Heriot-Watt University, Edinburgh, EHI 4 4AS, UK \\ †Laboratory for Information Photonics and Wave Signal Processing, Department of Information and Communication Engineering, \\ The University of Electro-Communications, I-5-I, Chofugaoka, Chofu, Tokyo, 182-8585, Japan \\ ‡Department of Photonics Engineering, DTU Fotonik, P. O. Box 49, DK- 4000 Roskilde, Denmark
}

\begin{abstract}
As an improvement of the intensity correlation used widely in conventional electronic speckle photography, we propose a new technique for displacement measurement based on correlating Stokes-like parameters derivatives for transformed speckle patterns. The method is based on a Riesz transform of the intensity speckle pattern, which converts the original real-valued signal into a complex signal. In closest analogy to the polarisation of a vector wave, the Stokes-like vector constructed from the spatial derivative of the generated complex signal has been applied for correlation. Experimental results are presented that demonstrate the validity and advantage of the proposed pseudo-Stokes vector correlation technique over conventional intensity correlation technique.
\end{abstract}

KEY WORDS: complex signal representation, displacement and strain measurement, pseudoStokes vector correlation, Riesz transform, speckle photography

\section{Introduction}

In experimental mechanics, the measurement of displacement and strain with high accuracy during complicated deformation of materials and devices continues to be a primary challenge [1]. Among various full field measurement techniques, digital image correlation (DIC) has received a lot of interests during the last decades due to its non-destructive nature and its relative ease of implementation and use [2-5]. Until now, the research in DIC has, for the vast majority, been based on the cross-correlation function of raw image intensity, and less attention has been paid to the complex field information associated with recorded image. Recently, we proposed the use of pseudo-phase information from the complex signal representation of image pattern generated by a Hilbert filtering and demonstrated the improved performance based on the pseudo-phase only correlation [6, 7]. Meanwhile, such transform can make full use of the available information from the recorded image, since new real, imaginary part, amplitude, and phase information have been introduced after the complex signal representation of a real-valued pattern.
The purpose of this paper is to propose yet another technique of speckle photography for displacement and strain measurement based on pseudo complex field information of the speckle patterns. Instead of directly correlating the raw intensity distributions of speckle patterns, we first generate the complex signal representation for a speckle pattern by Riesz filtering. Next, we extract the real and imaginary part of this generated complex field, and calculate the local gradient. Then we conduct the displacement and strain measurement by correlating these derivatives treated in a way, similar to the Stokes vectors used in polarised light. In fact, only three out of four Stokes components are independent, providing the basis for the cross correlation used to depict the displacement of the speckle pattern.

The use of the complex signal representation, and especially its derivatives, gives a sharper correlation function, as will later be shown. Furthermore, because the complex field can be obtained without recourse to interferometry, the technique can be applied to speckle-like artificial markings such as printed random dots and random textures of natural origin (e.g. biological tissues) under incoherent natural illumination. This versatility of the complex 
information representation opens up new possibilities in a wide range of applications beyond those known for laser speckle metrology. Although the complex field derived in this manner is not the real field of the laser speckle, this artificial complex field has a close relation to the underlying real-valued intensity pattern, and does provide useful information about the object-under-test. Because our technique is based on the cross-correlation of a Stokes-like vector in the spatial signal domain, we call it pseudoStokes vector correlation (PSVC) for complex signals to stress its difference from the conventional scalar correlation technique.

In the following, first we briefly review the complex analytic signal representation of a speckle pattern by Riesz filtering, and show how the real- and imaginary part can be extracted from the analytic signal. Based on this complex information, we conduct experiments of micro-displacement measurement by the proposed pseudo-Stokes technique to demonstrate the validity of the principle. Finally, we compare the proposed technique with the conventional techniques based on the raw intensity correlation, and demonstrate the advantage of the proposed PSVC.

\section{Principle}

\section{Complex signal representation of a speckle pattern}

Before explaining the proposed PSVC technique, we first review the complex signal presentation of a speckle pattern along the lines of our previous investigation [8].

In physics and in engineering, it is common practice to represent a real-valued signal by its associated complex signal representation [9]. The concept of an analytic signal, of which the real part is the original signal with its corresponding imaginary part being the Hilbert transform of the real part, was introduced to communication theory by Gabor in the 1940s [10]. Although the analytic signal has been widely adopted for one-dimensional (1-D) complex signal representations, an obvious anisotropy was found in the generated analytic signal when the Hilbert filter was applied to a 2-D speckle pattern, because the partial Hilbert filter introduces different spectral bandwidths along the $f_{x}$-and $f_{y}$-direction. Although the anisotropic analytic signal does not give rise to a bias or a scale change for the detected displacement, it results in a direction-dependent spatial resolution for the displacement measurement. To obtain a 2-D isotropic analytic signal of a 2-D speckle pattern, we have proposed to replace the partial Hilbert transform with the Riesz transform or vortex transform $[11,12]$.

Let $I(x, y)$ be the AC component of the original intensity distribution of the speckle pattern obtained after subtraction of the mean value (average DC component), and let its Fourier spectrum be $\Im\left(f_{x}, f_{y}\right)$. We can relate $I(x, y)$ to its isotropic analytic signal $\tilde{I}(x, y)$ through a Riesz filter. Thus, our definition of a 2-D isotropic complex signal representation for the speckle pattern is

$$
\begin{aligned}
\tilde{I}(x, y)= & \int_{-\infty}^{+\infty} \int_{-\infty}^{+\infty} V\left(f_{x}, f_{y}\right) \cdot \Im\left(f_{x}, f_{y}\right) \\
& \times \exp \left[j 2 \pi\left(f_{x} x+f_{y} y\right)\right] \mathrm{d} f_{x} \mathrm{~d} f_{y},
\end{aligned}
$$

where $V\left(f_{x}, f_{y}\right)$ is a spiral phase function with a vortex structure in the frequency domain defined as follows:

$V\left(f_{x}, f_{y}\right)=\frac{f_{x}+j f_{y}}{\sqrt{f_{x}^{2}+f_{y}^{2}}}=\exp \left[j \beta\left(f_{x}, f_{y}\right)\right]$,

with the phase $\beta \equiv \arctan \left(f_{y} / f_{x}\right)$ being the polar angle in the spatial frequency domain. As pointed out by Larkin et al. [12], the spiral phase function has the unique property that any section through the origin is a signum function with a $\pi$ phase jump. After straightforward algebra, we find

$$
\begin{aligned}
\tilde{I}(x, y) & =\operatorname{Re}\{\tilde{I}(x, y)\}+j \operatorname{Im}\{\tilde{I}(x, y)\} \\
& =|\tilde{I}(x, y)| \exp [j \theta(x, y)] \\
& =I(x, y) * v(x, y),
\end{aligned}
$$

where $\operatorname{Re}\{\cdots\}$ and $\operatorname{Im}\{\cdots\}$ represent the real and imaginary parts, * denotes the convolution operation, and $v(x, y)$ is the 2-D Riesz kernel,

$v(x, y)=\frac{j(x+j y)}{2 \pi\left(x^{2}+y^{2}\right)^{3 / 2}}=\frac{j \exp (j \alpha)}{2 \pi r^{2}}$

with $r \equiv \sqrt{x^{2}+y^{2}}, \alpha \equiv \arctan (y / x)$ being the distance and the azimuth angle in the spatial polar coordinates defined in the usual way.

Because the newly generated real and imaginary parts, amplitude and phase associated with the complex signal provide more effective means for analyzing, processing and understanding the available information from the recorded speckle pattern, the Riesz transform in Equation (1) effectively exploits the existing information, although the complex representation of a real-valued speckle pattern does not introduce new information. Furthermore, due to the fact that the newly generated real- and imaginary part can be detected without recourse to interferometry, the complex 
signal representation has the versatility that expands its applications beyond those known for laser speckle metrology, because it permits one to use general random patterns other than laser speckles. This includes a printed or projected random dot pattern or a random texture found on a natural object, here used for the purpose of micro-displacement, and strain measurement.

\section{Pseudo-Stokes vector correlation}

In this section, we will define and explain the proposed PSVC technique based on Stokes-like parameters associated with the complex signal representation of a speckle pattern. For the purpose of comparison, we also briefly outline the conventional techniques that use the intensity information of a speckle pattern.

At any position of the generated complex field $\tilde{I}$, its real and imaginary part can be locally approximated by

$\operatorname{Re}\{\tilde{I}(x, y)\}=a_{r} x+b_{r} y+c_{r}$,
$\operatorname{Im}\{\tilde{I}(x, y)\}=a_{i} x+b_{i} y+c_{i}$,

where the coefficients: $a_{k}, b_{k}, c_{k}(k=r, i)$ can be obtained by the least-square fitting method from the detected complex values at the pixel grid surrounding the observed position [8]. Therefore, the complex vector field $\nabla \tilde{I}$, which is the associated gradient of the complex signal representation $\tilde{I}$, can be expressed as

$$
\begin{aligned}
\nabla \tilde{I} & =\left(a_{r}+j a_{i}\right) \hat{x}+\left(b_{r}+j b_{i}\right) \hat{y} \\
& =\left(a_{r} \hat{x}+b_{r} \hat{y}\right)+j\left(a_{i} \hat{x}+b_{i} \hat{y}\right) .
\end{aligned}
$$

where, $\hat{x}$ and $\hat{y}$ are unit vectors. Recently, a set of Stokes-like parameters has been proposed for the complex vector field $\nabla \tilde{I}$ that shares similar geometric features associated with the vector polarisation field [13-15]. Following this, the parameters, describing the local gradient of the complex signal representation of a 2-D speckle pattern, may be defined as:

$$
\begin{aligned}
& S_{0}=a_{r}^{2}+b_{r}^{2}+a_{i}^{2}+b_{i}^{2}, \\
& S_{1}=a_{r}^{2}+a_{i}^{2}-b_{r}^{2}-b_{i}^{2}, \\
& S_{2}=2\left(a_{r} b_{r}+a_{i} b_{i}\right), \\
& S_{3}=2\left(a_{r} b_{i}-a_{i} b_{r}\right) .
\end{aligned}
$$

These parameters describing the anisotropy related to $\nabla \tilde{I}$, are mathematically analogous, but physically unrelated, to the Stokes parameters in polarisation optics. From Equation (8), it is easily found that only three of them are independent since they are interrelated by the identity:

$S_{o}^{2}=S_{1}^{2}+S_{2}^{2}+S_{3}^{2}$

Following the same procedure as used for polarisation [16], we can represent the local gradient of the complex signal representation $\nabla \tilde{I}$ as a Stokes-like vector: $\vec{S}=\left(S_{1}, S_{2}, S_{3}\right)$. The vector $\vec{S}(x, y)$ of the complex signal representation of a speckle pattern can be referred to as the pseudo-Stokes vector to distinguish it from the Stokes description used widely for polarisation of a vector electric field.

Usually, the in-plane displacement of an object can be detected by the cross-covariance of the intensity information of a speckle pattern observed on the object, which is defined by

$C_{I}(\Delta x, \Delta y)=\left|\left\langle I^{\prime}(x+\Delta x, y+\Delta y) I(x, y)\right\rangle\right|$,

where $I(x, y)$ and $I^{\prime}(x, y)$ are the AC-components of the intensity distribution of speckle pattern before and after the displacement, respectively, which are obtained after the mean values are subtracted, and angular brackets $\langle\cdots\rangle$ indicates the ensemble average. Therefore, the in-plane displacement of the object can be measured from the position of the crosscovariance peak.

Instead of the conventional intensity correlation function for deriving the speckle displacement, here, we propose the PSVC. That is

$$
\begin{aligned}
C_{\mathrm{PSVC}} \equiv & \left|\left\langle\vec{S}^{\prime}(x+\Delta x, y+\Delta y) \cdot \vec{S}(x, y)\right\rangle\right| \\
= & \mid\left\langle S_{1}^{\prime}(x+\Delta x, y+\Delta y) S_{1}(x, y)\right\rangle \\
& +\left\langle S_{2}^{\prime}(x+\Delta x, y+\Delta y) S_{2}(x, y)\right\rangle \\
& +\left\langle S_{3}^{\prime}(x+\Delta x, y+\Delta y) S_{3}(x, y)\right\rangle \mid .
\end{aligned}
$$

Before the end of this session, several considerations have to be addressed with respect to this more cumbersome way for the speckle displacement measurement. There is no doubt that the use of the PSVC technique calls for longer processing time as compared with the intensity correlation technique, since the pseudo-Stokes vector should be constructed from the complex signal representation of a speckle pattern before the correlation operation. Despite the annoying time consumption, the proposed PSVC do 
have several unique properties as compared with the conventional intensity correlation. Firstly, the result of essentially correlating the first derivative of the speckle pattern as compared with the speckle pattern, itself, results in a decreased width of the correlation function. This in turn, will increase the accuracy of the method. One has to remember that the radius of curvature of the correlation peak is inversely proportional to the normalised variance of the power spectrum [17]. On the other hand, it is well known that differentiation of a speckle pattern is prone to increasing the influence of the noise, and therefore, giving rise to erroneous peaks in the correlation function. While, the proposed PSVC will severely reduce this effect - even reducing the number of side lobes in the correlation function, as will be demonstrated in the next session.

\section{Experiments}

We conducted a set of simple experiments where a rough surface was illuminated with a collimated laser beam, and the scattered light was recorded by a CCD image sensor. By acquiring two images of the speckle patterns before and after a minor controlled surface displacement, the local displacement was determined using the two techniques, viz. the IC- and the PSVCtechnique. Figure 1 shows an example of the recorded speckle pattern, and its corresponding distributions of the pseudo-Stokes parameters to be used for the displacement measurement. Just as the well-known granular structure for the intensity of laser speckle pattern, each component of the gener-
(A)

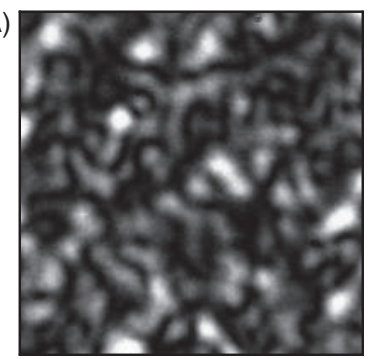

(C)

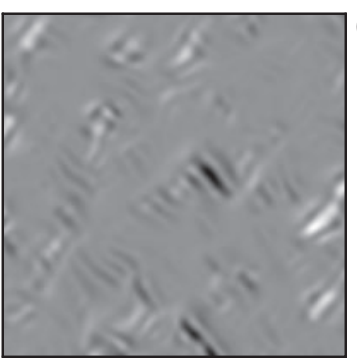

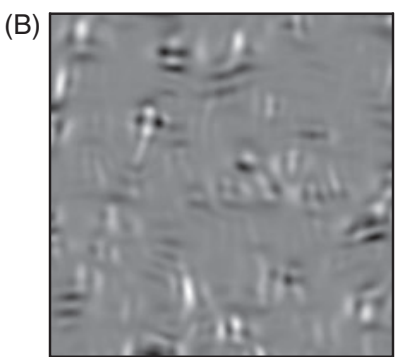

(D)

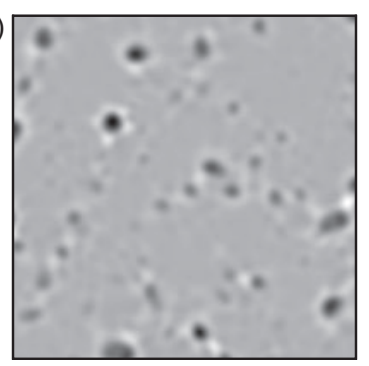

Figure I: Various representations of the speckle field. Original speckle pattern (A), and the associated pseudo-Stokes parameters: $S_{1}(\mathrm{~B}), S_{2}(\mathrm{C})$ and $S_{3}(\mathrm{D})$ ated pseudo-Stokes vector also has the desired random structures. It is the randomness of the pseudoStokes parameters that enable the application of the vector correlation.

The modulus of the normalised cross covariance functions for intensity correlation (IC) and PSCV are illustrated in Figure 2 with the sub-window size of $100 \times 100$ pixels (a) and $20 \times 20$ pixels (b). As shown in Figure 2, two essential issues have been demonstrated for the performance comparison between IC and PSCV. Firstly, the width of the main peak for the cross-covariance is decreased for PSVC, thus increasing the resolution of the measurement. Secondly, the size of the side lobes is decreased as well. The main thrust for the proposed PSCV technique is the possibility for dividing the observed area up into sub-areas in which the relative in-plane displacement can be followed. This will provide a tool for analyzing the relative displacement for a larger area with a given but finite number of pixels. To fulfil this task, the efficiency of this method for smaller interrogation
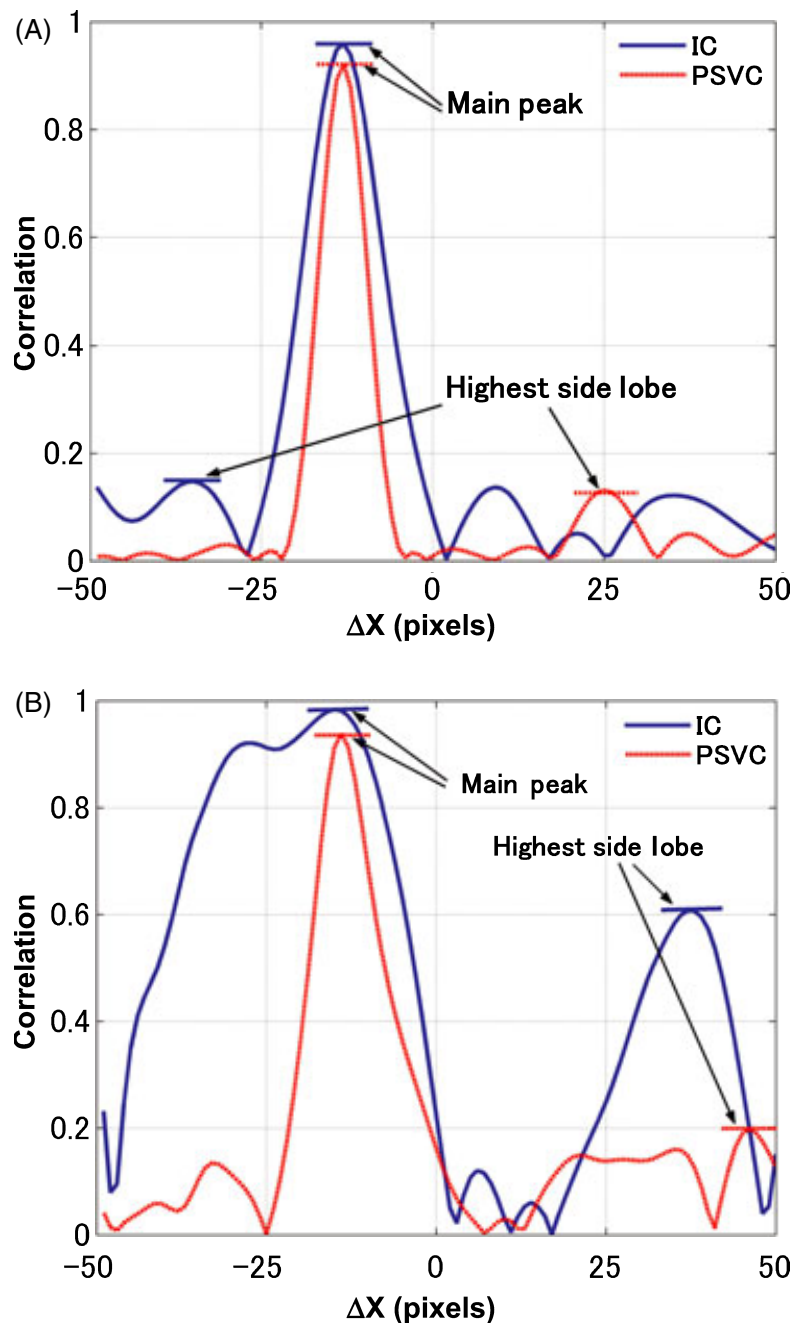

Figure 2: Modulus of cross-covariance functions for intensity correlation and for PSVC with a correlation window size of $100 \times 100$ pixels $(\mathrm{A})$, and $20 \times 20$ pixels $(\mathrm{B})$ 

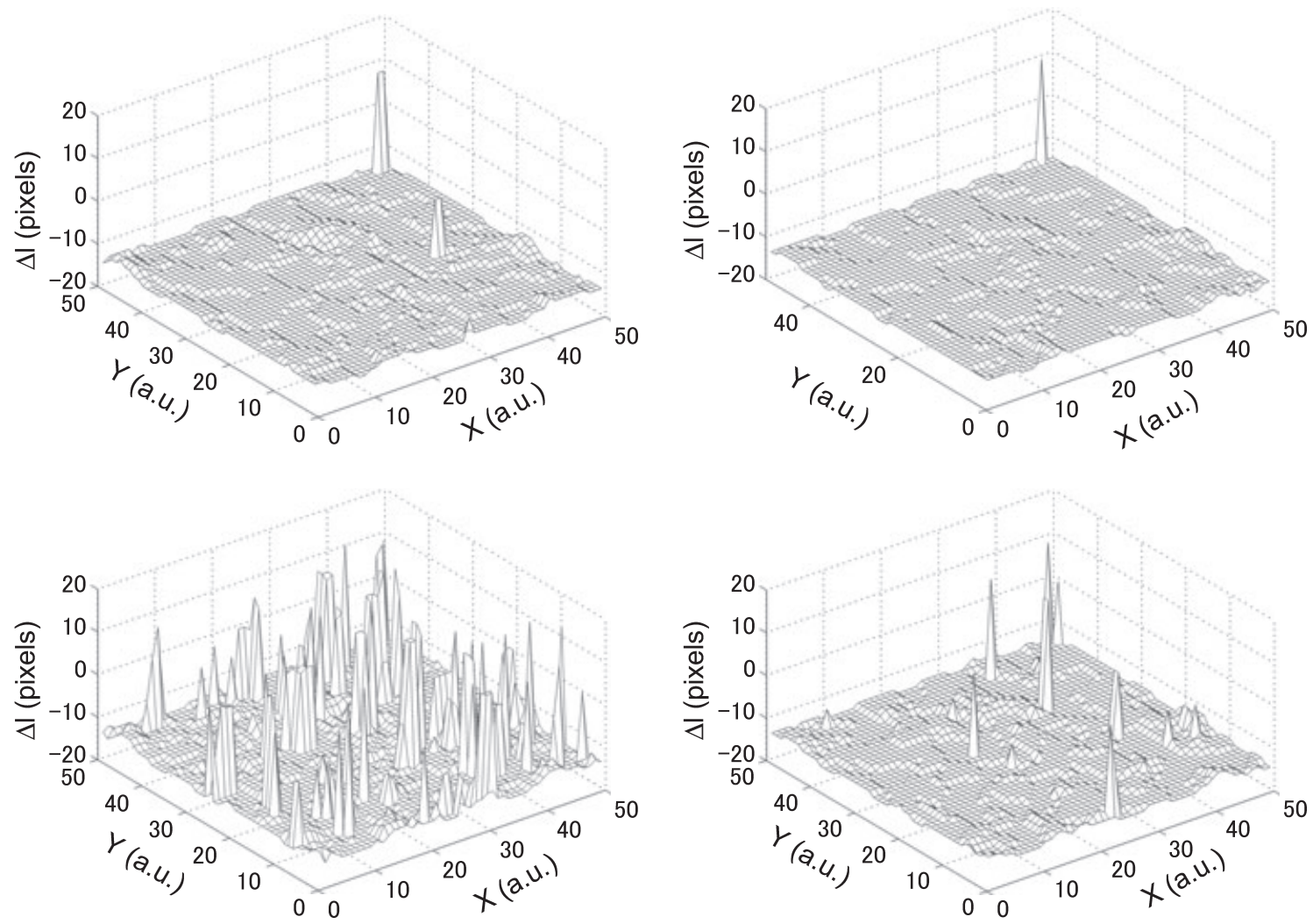

Figure 3: Three-dimensional view of measured uniform displacement. Upper row is for a correlation window size of $100 \times 100$ pixels; lower row is for a correlation window size of $20 \times 20$ pixels; left column is given by intensity correlation; and right column is by PSVC

areas measured in pixels has to be addressed. Figure 3 shows three-dimensional plots of the spatial measurement for a uniform displacement, where the $z$ axis indicates the local displacement $\Delta l$ in unit of pixels at the corresponding position $(x, y)$ on the sample's surface. By estimating the peak position of the crosscovariance, pixel by pixel, the correct displacement of -14 pixels has been found for the majority of spatial positions. As expected, it can be easily observed from Figure 3 that decreasing the number of pixels used for the correlation will increase the number of possible measurement errors indicated by many local peaks on the surface. But it is evident that PSVC outperforms IC with respect to suppressing incorrect side lobe for better determination of the correct covariance peaks.

To quantify the above statements, a series of tests were performed with varying window size. As shown in Figure 4(A) for the strength of the spurious side lobes, the suppression of secondary lobes given by PSVC is almost a factor of two better than for IC, independent of the window size. Figure $4(\mathrm{~B})$ is the root mean square error defined by

$$
\mathrm{RMSE}=\sqrt{\sum_{i=1}^{N}\left[\left(\Delta x_{i}-\overline{\Delta x}\right)^{2}+\left(\Delta y_{i}-\overline{\Delta y}\right)^{2}\right] / N}
$$

where $(\overline{\Delta x}, \overline{\Delta y})$ are the average values for the measured displacement, and $N$ is the total number of pixels in the correlation window. The root mean square error for displacement estimation is reduced by a factor of three for smaller windows using PSVC; but for larger windows, the two processing schemes end up having the same accuracy.

\section{Conclusions}

We have proposed a new technique for displacement measurement, referred to as PSVC. The method is based on recording a speckle pattern before and after a displacement of the scattering object. The displacement of the speckle pattern usually reveals the strain of test samples subject to in-plane displacement or two out-of-plane angular displacements, i.e. torsion. The recorded imagery is transformed into a complex field by a Riesz transform, and the spatial derivatives have been applied to give four parameters for each pixel. The combination of these four parameters shares the formal analogy to complex vector field, and can be constructed as the Stokes-like parameters used in dealing with polarised light. The Stokes' parameters before and after a displacement are cross correlated, and the peak position reveals 

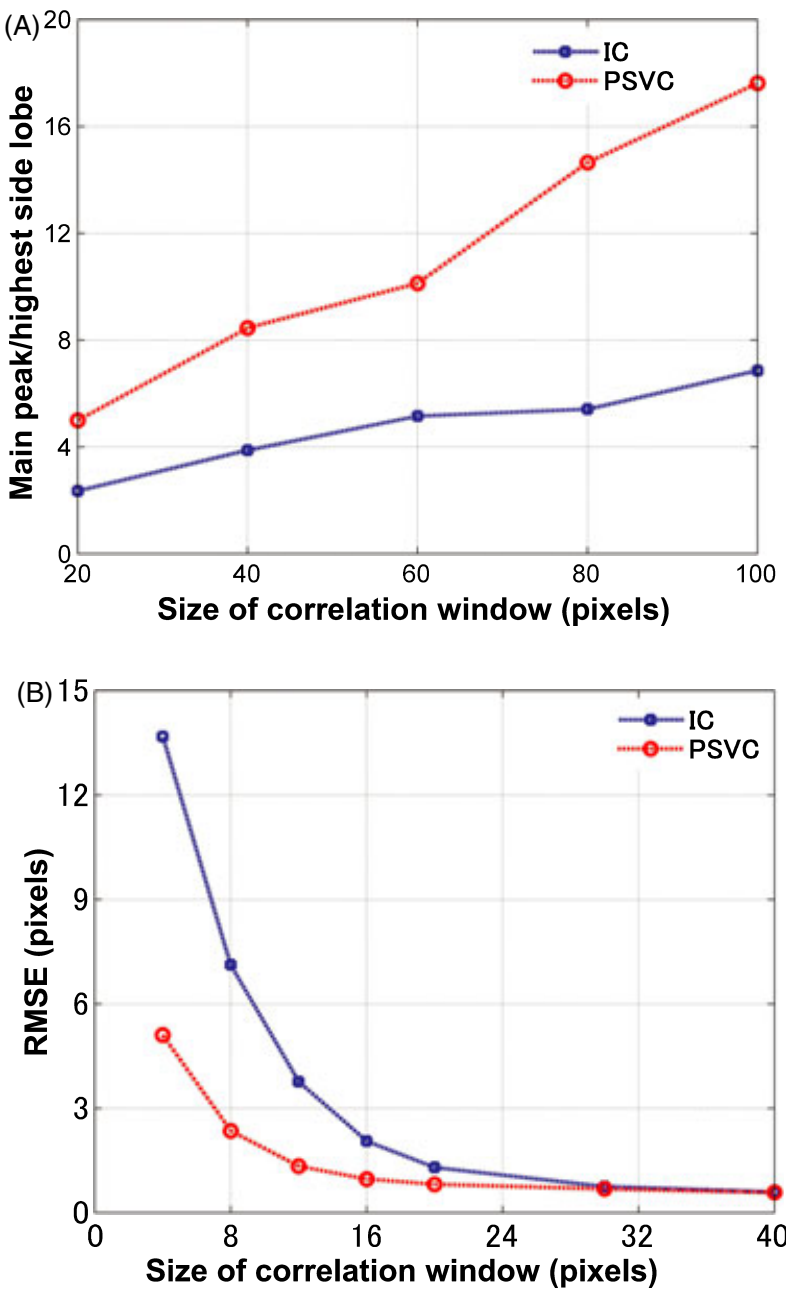

Figure 4: The fraction of the main peak covariance height to the highest side lobe as a function of the window size (A); and the root mean square error measured in pixels as a function of the window size (B)

the displacement of the speckle within the correlation window. The method, although being a little more time-consuming, has three advantages: First, the width of the correlation peak is reduced, giving rise to a higher spatial resolution and accuracy in displacement determination. Secondly, the suppression of secondary peaks in the correlation function is increased, reducing the risk of locking on to an erroneous peak, and avoiding the usually associated increase of noise by correlating the full information of the derivatives in two dimensions for both the real- and the imaginary parts of the Riesz filtered speckle pattern. Finally, the transform of the spatial derivatives to a Stokes-like vector makes the identification less prone to decorrelation of the speckle pattern suffered between two recordings, and further suppress spurious peaks in the correlation plane. Experiments have been performed that demonstrate the advantage of the proposed PSVC as compared with the conventional technique based on intensity correlation.

\section{ACKNOWLEDGEMENTS}

Part of this work was supported by Grant-in-Aid of JSPS B (21360028), and by The 21st Century Center of Excellence (COE) Program on 'Innovation of Coherent Optical Science' granted to The University of Electro-Communications. One of the authors greatly acknowledges the financial support from the Danish Council for Technology and Innovation under the Innovation Consortium CINO (Centre for Industrial Nano Optics).

\section{REFERENCES}

1. Sirohi, R. S. (1993) Speckle Metrology. Marcel Dekker Inc., New York.

2. Chu, T. C., Ranson, W. F., Sutton, M. A. and Peters, W. H. (1985) Applications of digital image correlation techniques to experimental mechanics. Exp. Mech. 25, 232-244.

3. Chen, D. J., Chiang, F. P., Tan, Y. S. and Don, H. S. (1993) Digital speckle displacement measurement using a complex spectrum method. Appl. Opt. 32, 1839-1849.

4. Sjödahl, M. and Benckert, L. R. (1993) Electronic speckle photography: analysis of an algorithm giving the displacement with subpixel accuracy. Appl. Opt. 32, 22782284.

5. Sjödahl, M. (1994) Electronic speckle photography: increased accuracy by nonintegral pixel shifting. Appl. Opt. 38, 6667-6673.

6. Wang, W., Ishii, N., Hanson, S. G., Miyamoto, Y. and Takeda, M. (2005) Pseudophase information from the complex analytic signal of speckle fields and its applications. Part I: Micro-displacement observation based on phase-only correlation in the signal domain. Appl. Opt. 44, 4916-4921.

7. Wang, W., Ishii, N., Hanson, S. G., Miyamoto, Y. and Takeda, M. (2005) Pseudophase information from the complex analytic signal of speckle fields and its applications. Part II: Statistical properties of the analytic signal of a white-light speckle pattern applied to the microdisplacement measurement. Appl. Opt. 44, 4916-4921.

8. Wang, W., Yokozeki, T., Ishijima, R., Wada, A., Miyamoto, Y., Takeda, M. and Hanson, S. G. (2006) Optical vortex metrology for nanometric speckle displacement measurement. Opt. Exp. 14, 120-127.

9. Goodman, J. W. (1985) Statistical Optics. John Wiley \& Sons, New York.

10. Gabor, D. (1946) Theory of communications. J. IEE 93, $429-457$.

11. Riesz, M. (1927) Sur les functions conjuguees. Math. Zeitschrift 27, 218-244.

12. Larkin, K. G., Bone, D. J. and Oldfield, M. A. (2001) Natural demodulation of two-dimensional fringe patterns. I. General background of the spiral phase quadrature transform. J. Opt. Soc. Am. A 18, 1862-1870.

13. Dennis, M. R. (2004) Local structure of wave dislocation lines: twist and twirl. J. Opt. A: Pure Appl. Opt. 6, s202s208.

14. Egorov, Y. A., Fadeyeva, T. A. and Volyar, A. V. (2004) The fine structure of singular beams in crystals: colours and polarization. J. Opt. A: Pure Appl. Opt. 6, s217-s228. 
15. Wang, W., Dennis, M. R., Ishijima, R., Yokozeki, T., Matsuda, A., Hanson, S. G. and Takeda, M. (2007) Poincaré sphere representation for the anisotropy of phase singularities and its applications to optical vortex metrology for fluid mechanical analysis. Opt. Exp. 15, 11008-11019.
16. Born, M. and Wolf, E. (1999) Principles of Optics, Cambridge University Press, Cambridge.

17. Beck, M. S. and Plaskowski, A. (1987) Cross Correlation Flowmeters: Their Design and Application, Adam Hilger, Bristol. 